\title{
The clinical day at CARS: philosophy, strategy, and the future
}

\author{
Leonard Berliner $^{1}$ (D) Eric vanSonnenberg ${ }^{2} \cdot$ Hubertus Feusner $^{3} \cdot$ Dirk Wilhelm $^{3} \cdot$ Heinz U. Lemke $^{4,5}$
}

Received: 6 November 2021 / Accepted: 26 January 2022 / Published online: 18 February 2022

(c) CARS 2022

\begin{abstract}
Purpose For over three decades, the Computer-Assisted Radiology and Surgery (CARS) International Congress and Exhibition has provided a forum for the presentation of innovations in computer applications in radiology, medicine, and surgery. A unique feature of the CARS meetings is the interplay between scientists, engineers, and physicians. Since 2007, a Clinical Day program was introduced to the Congress highlighting the practical applications of new technology within the context of clinical medical and surgical practice.

Methods The Clinical Day of the CARS Congress allows cross-fertilization of ideas between technologically oriented engineers and clinically oriented physicians; two groups who typically have little interaction. Activities of the Clinical Day include presentations by invited speakers, presentations of Innovative Clinical Investigations, a Panel Discussion and Open Forum, and, most recently, real-time clinical presentations with professionally prepared scholarly videos. Special consideration is given to young researchers and students. There has been an explosion of interest in the Clinical Day with continued and growing interest in Artificial Intelligence, Computer-Assisted Surgery, and new scientific breakthroughs as they become linked to clinical applications.

Results Success of the Clinical Day is emphasized by increased participation and efforts to expand the scope and depth of Clinical Day activities. The Open Forum has proven to be highly effective in identifying important new technologic challenges in medicine and promoting discussion among those whose expertise likely can lead to solutions.

Conclusions The original goal of the Clinical Day, to provide an effective means to "bridge the gap" between the engineering community and practicing physicians and surgeons, has been realized through the presentation and discussion of real-life, clinical material that utilizes advanced technology. The program has served to inspire young researchers by allowing them to see the end results of their laboratory investigations, thereby gaining a greater appreciation of the importance of their work.
\end{abstract}

Keywords Computer-assisted radiology and surgery $\cdot$ Clinical day $\cdot$ Conferences, computer-assisted surgery $\cdot$ Conferences, computer-assisted radiology $\cdot$ Conferences, biomedical engineering

\section{Introduction}

The initial Clinical Day session at CARS took place at the 21st International Congress and Exhibition, in Berlin Ger-

Leonard Berliner

lberliner1@northwell.edu

1 Department of Radiology, Staten Island University Hospital Northwell Health, Staten Island, NY, USA

2 Department of Radiology, University of Arizona College of Medicine - Phoenix, Phoenix, AZ, USA

3 Department of Surgery, Klinikum Rechts Der Isar der Technischen Universität München, Munich, Germany

4 International Foundation for CARS, Kuessaberg, Germany

5 Department of Radiology, University of Southern California, Los Angeles, USA many, June 27-30, 2007. The concept of a Clinical Day had been percolating for several years and was practiced in joint sessions in dialog form between clinicians and engineers. The Clinical Day program has evolved to become a valuable and standard part of CARS meetings.

However, in 2020 and 2021, the nature of the Clinical Day was altered by the transformation of the CARS Conference into a "hybrid" meeting that included both live and virtual participation due to the COVID-19 pandemic. After a considerable amount of planning and effort, the Clinical Day at the 35th International Congress and Exhibition in Munich (CARS 2021) included: (1) Interactive Live Sessions from robotic surgeries, (2) a presentation from the MRI Research Laboratory; (3) presentations from industry; (4) the DICOM Working Group 24 meeting; and (5) a Closing Session, that 
included an open forum on the importance of the Clinical Day, and what it means to young investigators.

This editorial affords the opportunity to present our thoughts and philosophy regarding the CARS Clinical Day, now, and for the foreseeable future. Herein, we review important material that rarely gets discussed formally during the Clinical Day. We will try to recall the personal reflections, outlooks, philosophies, and aspirations of CARS participants that often are discussed informally at dinners, coffee breaks, and at social events. While the participants may feel that their opinions begin and end in these casual conversations, in reality, many of these concepts and proposals are seized by members of the CARS Organizing Committee at its meetings. Serious consideration is given to these ideas that, over the years, have stimulated substantial change and improvement in the CARS meetings.

We will present our impression of CARS conferences and the origins of the Clinical Day Program. We will explore how the Clinical Day has evolved and expanded to include more participants and new topics, such as Artificial Intelligence (AI). We are always attempting to broaden and enrich the Domains of Discourse of CARS!

\section{Origins}

The CARS conferences, if not unique, certainly are unusual; undoubtedly that is by the design of many members of the CARS Congress Organizing Committees throughout the years. The integration of scientists, engineers, and physicians, while seemingly is disparate, but on the contrary, is a marvelous means to cross-fertilize ideas, to integrate with those whom we physicians typically would have little interaction, and has been a golden opportunity to identify our technologic challenges with the purpose of sharing them with those whose expertise likely can lead to solutions. Once we, as faculty, and of course the attendees, have realized that CARS is not like typical medical conferences, worlds open up to hone in on issues that pique the interest of engineers and scientists who use their expertise to solve clinical patient care issues.

Typical examples can be cited here from earlier CARS Congresses in the 1980s, when Computer Aided Detection (CAD) [1] and Computer-Assisted Surgery (CAS) [2] evolved from the interdisciplinary cooperation of engineers, radiologists, and surgeons, setting the stage for what is considered today to be state of the art. Many of the foundational concepts of CAD and CAS were presented jointly in dialog form by engineers and clinicians at CARS congresses.

The Clinical Day began with a philosophy and a strategy. The philosophical goal was to "bridge the gap" between the engineering community and surgeons through the presentation of real-life, clinical material that utilized advanced technology. The intention was to inspire young researchers by giving them the opportunity to see the end results of their laboratory investigations, thereby gaining a greater appreciation of the importance of their work. The initial strategy involved providing the CARS meetings with a platform to increase exposure to both Interventional Radiology and Interventional Oncology. Many of the early Clinical Day sessions were devoted to the techniques and technologies of Interventional Radiology by radiologists who performed tumor ablation. Within a year or two, the scope of the Clinical Day expanded to include other minimally invasive procedures, specifically interventional bronchoscopy and interventional endoscopy, and complex imaging technologies such as PETCT for guiding therapies. Wherever possible, guest speakers were invited from active CARS participants and local sponsoring universities, as well as other luminary sources, such as the NIH. Experts who were acknowledged in their fields were recruited to provide the newly introduced annual "Stanley Baum Lecture." There has been an explosion of interest in the Clinical Day, due to a wide variety of factors including the continued and growing interest in AI, CAS, and new scientific disciplines as they become linked to clinical applications. Thus, the doors were opened to all disciplines, such as neurosurgery, interventional cardiology, cardiac surgery, and orthopedics. The program was further augmented with the inclusion of peer-reviewed abstracts that have been identified as "Innovative Clinical Investigations."

\section{Young researchers}

The enthusiasm seen in younger CARS participants confirmed the importance of reaching out to universities, institutions, and laboratories to provide students and research assistants with a vision of the clinical and practical end results of their efforts. These individuals who attend CARS already have achieved some degree of success and recognition in their fields, as most attendees have had papers and/or posters accepted for presentation. Thus, it is natural to reach out to students and researchers in biomedical engineering and related disciplines, drawing them into discussions regarding the involvement of the next generation to shape the future of advanced medical, surgical, and imaging technology.

A common theme expressed by young researchers is that they have learned techniques and technologies and are now looking for meaningful projects. They find it challenging to connect with physicians and hospitals; specifically, they encounter restrictions in access to patient data and images due to privacy issues. They are seeking guidance to learn what types of new tools and algorithms are required by the medical/surgical community. We have made efforts in the Clinical Day presentations to highlight relevant items for 
them to address in their laboratories and to discuss their concerns at the coffee breaks, receptions, and social events.

\section{Medical students}

There is an increasing awareness of the nexus and potential important opportunities that involve engineering majors who have entered medical school. Several US medical schools have started a formal integration between educational engineering institutions and medical schools.

To further gain insights and to expand the nexus, we, at the University of Arizona College of Medicine Phoenix, are engaged in three projects on the subject: (1) analyzing the backgrounds and profiles of college engineering majors who matriculate into medical school, (2) attitudes and challenges in medical school for these students, and (3) what paths engineering majors pursue after graduation from medical school.

We also have medical students engaged in numerous projects related to AI as it pertains to medical school, and its integration into clinical medical practice. Numerous publications on AI by these engineering majors who have become medical students have occurred.

During the past several years, we have begun to report on these activities during the Clinical Day program. It is our goal to explore and share educational pathways by reaching out to medical students to find ways to raise awareness and involvement in medical engineering and AI. We have had two national/international Medical Student Radiology Symposia and $\mathrm{AI}$ has been included. We also have started an AI Interest Group for medical students.

\section{Panel discussion/open forum}

In recent years, the "Panel Discussion" has been transformed into an "Open Forum," in which all audience attendees, along with the Panel members, are encouraged to express their ideas, as well as their vision for the present and the future of medicine. The goal of the Open Forum is to achieve a blend of the wisdom and experience of the senior participants with the innovative ideas and energy of the younger participants.

The spectrum of backgrounds of CARS faculty, as well as the diversity of the conference attendees, makes for a perfect setting to discuss current challenges in medicine and its attendant technology and potential solutions. Encouraging visionary solutions to improve patient care is a goal for young scientists. The amalgamation of scientists, engineers, and clinical physicians repeatedly has made for lively, innovative, and avant-garde discussions during these panel sessions.

\section{Real-time demonstrations/toward improving patient care}

One might ask what benefit and relevance a Clinical Day has at a time when video has advanced as the general and omnipresent communication standard for scientific innovations. Clinical advances and the results of ongoing research are now available on video channels that are accessible to everyone. Technologic problems and innovative research have reached quasi-molecular dimensions, beyond typical clinical practitioners. What is the sense and significance behind a report from everyday clinical practice when the development cycles have become so short that what we consider an innovation today will be outdated soon thereafter?

Computer-assisted medicine, albeit technologic, must remain patient-centric. In all medical fields, the patient, the treating physician, and the work environment should be the center of all efforts and must remain the focus. Research should never be an application of technology for its own sake, but always must be focused on clinical requirements for the improvement of medical care.

At a time when specialization and diversification are continually increasing, not only in medicine but also in technical disciplines, refocusing on this common goal might be more relevant than ever. This can be achieved by looking into the clinics and over the shoulders of those treating patients. By understanding everyday activities, especially clinical needs and shortcomings, and with technologic advances, the goal of improved patient care can be achieved, and the scientific spirit of CARS members should be further enhanced.

The Clinical Day may not be restricted only to procedures considered "standard of care," but should include cutting edge technology and innovations that facilitate improved patient care. To present at the Clinical Day should become the highest goal and motivation for young scientists. For a biomedical researcher of any age, being able to present one's investigative work that has been transformed into a clinically valuable product is one of the highest forms of recognition and success that may be achieved. One may feel as an individual who has entered the "champions league of innovative research"! The Clinical Day provides the forum to present such clinically innovative work, which in its finest form may be thought of as the application of defined technology, in a sequence of well-aligned processes, akin to a well-orchestrated symphony. The Clinical Day is uniquely poised to allow presentation of these clinical innovations in their fully complexity, that may resemble a mosaic of wellintegrated components. By doing so, the Clinical Day can serve as a catalyst to recalibrate our research by precisely aiming our endeavors at the appropriate clinically critical targets.

In contrast to providing typical scientific presentations, the Clinical Day might allow presentations by teams of 
investigators, rather than by individuals alone. Thus, we can combine the work of engineers, physicians, and industrial partners. Since industrial affiliations in science often are viewed with suspicion, there is a need for open disclosure to avoid the appearance of a conflict of interest. However, it should be remembered that Industry, in many ways, fosters development and drives clinically oriented scientific research. Industrial support is indispensable for aspects of scientific conferences, but are subject to restrictions. Thus, the Clinical Day serves as a perfect venue to forge harmony among those who contribute to the translation of innovative research to clinically applied solutions. It addresses not only scientific questions, but also provides opportunity for discussion of topics that philosophically and ideologically serve as background for innovative concepts like Surgineering [3] - defined as the interactive and collaborative efforts of surgeons and engineers to conceptualize, develop, and produce the most clinically accurate and effective surgical tools.

\section{Vision on the future of medicine and the role of the clinical day}

It can be expected that the complexities of designing the OR, hospital, and health care systems of the future will increase beyond the capabilities of any specific professional expertise or group. To be prepared for this development would require extending the Clinical Day to include experts not only from the CARS community, but also from architecture, hospital administration, economics, and social sciences [4]. Their participation would allow cross-pollination of the various disciplines, thereby promoting mutual awareness and understanding of their respective roles regarding the "iron triangle of health care," i.e., quality, costs and access for all. Thus, discrepancies in the envisaged concepts and priorities of these various stake-holders may be reduced.

Questions and topics that should be addressed in these future Clinical Day settings could include:

1. Relative role of the primary variables such as quality, costs, and access for all, in decision-making on CARS $\mathrm{R} \& \mathrm{D}$, and the development and deployment of computerassisted medical systems.

2. Identification of significant secondary variables, such as patient involvement, clinician workflow, specific CARS technologies, and, the initial guiding themes of the CARS conferences, as well as CARS Foundation: Innovative, Interdisciplinary, and International $\left(I^{3}\right)$.

3. Confounding variables such as statistically low frequency events or causal indeterminable impact factors in the OR (e.g.- - team spirit), hospital (e.g.- transparency in decision making), health care system (e.g.- - health awareness), or society at large (e.g.-cultural differences).

4. Current patient expectations, as well as expectations for the future, that takes into consideration the geographic and cultural factors that have profound influence on the delivery of health care.

5. Role of disease prevention and Precision Medicine and their possible impact, as well as the role of CARS innovations in their development and implementation.

6. Promoting a sense of well-being and accomplishment among health care professionals, students, and researchers.

An important aspect of the Clinical Day, within a hybrid CARS conference, will be to present the questions and possible answers, not only in an informative manner, but at the same time making use of effective communication to inform, persuade, and entertain. This was very effectively demonstrated in the Clinical Day at CARS 2021, with professionally well-prepared scholarly videos capable of captivating the audience for the entire day.

\section{Innovations for the clinical day}

To realize the many themes of CARS, the spirit of the Clinical Day should not be limited to a specific day, but be incorporated each day, and, if appropriate, be included in a Clinical Hour at the end of particular CARS congress days. In addition, clinically important presentations throughout the meeting may, in the future, be designated as part of a "Clinical Day Track" in the conference program. The effectiveness of this type of extension of the Clinical Day throughout the conference was demonstrated at the CARS 2021 meeting. Furthermore, the virtual components allowed greater participation in the meeting, while reaching a broader audience, and will likely remain as an important and integral part of each year's program.

Session chairs could summarize and discuss the potential clinical impact of what has been presented that day, motivating the "Next Generation of Scientists" to reflect on the content of the research and the eventual application of this research into clinical practice.

To publish these insights in IJCARS, a new publication category, "Innovative Clinical Investigations," has been introduced. In general, these types of manuscripts focus on how new functionalities provided by IT tools and systems can be incorporated into the practice of medicine, specifically the disciplines of Radiology and Surgery. Within the review process of IJCARS, authors are encouraged to address questions related to the impact of their innovation on diagnostic/therapeutic workflows, i.e.- - what is the practical application into clinical medicine? Authors should 
indicate how these methods/tools potentially impact clinical outcomes for patients to improve health care. These questions represent the grand challenges for the Next Generation of Scientists that CARS conferences can illuminate.

\section{Conclusion}

For more than 35 years, the CARS Conferences have been dedicated to finding innovative solutions to the complexities of medical/surgical challenges, through the application of technology. Physicians, researchers, university laboratories, and departments of research and development in industry have different perspectives, resources, areas of expertise, and core competencies. The Clinical Day Open Forum has provided an opportunity for all CARS participants to discuss and share their views and visions of the past, present, and future of medicine and surgery. The Clinical Day has become a paradigm of the integration of clinical engineering and scientific expertise to produce solutions for practical clinical problems for the betterment of patients. This is truly in keeping with the two mantras adopted by this year's CARS Congress, found in this year's CARS Program announcement: "Next Generation of Scientists at CARS" and "Rethinking the Hospital and the OR."

It is the hope of the authors that we have conveyed the sense of importance of the Clinical Day at the CARS conferences. We have tried to effectively share our vision of how the Clinical Day can unite medical and surgical practitioners, researchers, engineers, students, and Industry to fulfill the promises for a better future that modern health care technology can provide.

Author Contributions All authors contributed to the conception, design, and/or content of this Editorial. The first draft of the manuscript was written by Leonard Berliner and all authors commented on previous versions of the manuscript and provided their additions to the manuscript. All authors read and approved the final manuscript.
Funding No funding was received to assist with the preparation of this manuscript. No funds, grants, or other support were received for producing this Editorial.

\section{Declarations}

Conflict of interest The authors did not receive support from any organization for the submitted work.

\section{References}

1. MacMahon H, Doi K, Giger ML, Katsuragawa S, Nakamori N, Sasaki Y (1989) Artificial intelligence in chest radiology: computeraided detection and quantitation of disease. In: Proc International Symposium CAR 89. Springer, Berlin Heidelberg New York Tokyo, pp 441-451

2. Moesges R, Schloendorff G, Klimek L, Meyer-Ebrecht D, Krybus W, Adams L (1989) CAS- Computer assisted surgery. An innovative surgical technique in clinical routine. In: Proc International Symposium CAR 89. Springer, Berlin Heidelberg New York Tokyo, pp 413-415

3. Feussner H, Wilhelm D, Navab N, Knoll A, Lüth T (2019) Surgineering: a new type of collaboration among surgeons and engineers. Int J Comput Assist Radiol Surg 14(2):187-190

4. Niederlag W, Lemke HU, Nagel E, Dössel O (eds) (2008) Vol 12. Gesundheitswesen 2025, Health Academy. Dresden, Germany.

Publisher's Note Springer Nature remains neutral with regard to jurisdictional claims in published maps and institutional affiliations. 